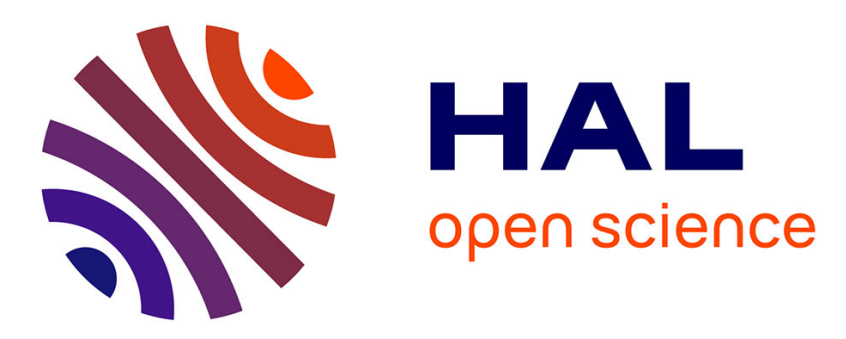

\title{
Study of the Oscillation Condition of Quartz Oscillators by Gyrator Transformation
}

\author{
Nicolas Ratier, Mahmoud Addouche, Daniel Gillet, Rémi Brendel, Jérome
}

Delporte

\section{- To cite this version:}

Nicolas Ratier, Mahmoud Addouche, Daniel Gillet, Rémi Brendel, Jérome Delporte. Study of the Oscillation Condition of Quartz Oscillators by Gyrator Transformation. IEEE/ EIA International Frequency Control Symposium, Jun 2000, Kansas City, Kansas, United States. pp.455-458. hal00345194

\author{
HAL Id: hal-00345194 \\ https://hal.science/hal-00345194
}

Submitted on 8 Dec 2008

HAL is a multi-disciplinary open access archive for the deposit and dissemination of scientific research documents, whether they are published or not. The documents may come from teaching and research institutions in France or abroad, or from public or private research centers.
L'archive ouverte pluridisciplinaire HAL, est destinée au dépôt et à la diffusion de documents scientifiques de niveau recherche, publiés ou non, émanant des établissements d'enseignement et de recherche français ou étrangers, des laboratoires publics ou privés. 


\title{
STUDY OF THE OSCILLATION CONDITION OF QUARTZ OSCILLATORS BY GYRATOR TRANSFORMATION
}

\author{
N. Ratier, M. Addouche, D. Gillet, R. Brendel, J. Delporte $\dagger$ \\ Laboratoire de Physique et Métrologie des Oscillateurs, Besançon, France* \\ Centre National d'Etudes Spatiales, Toulouse, France ${ }^{\dagger}$
}

\begin{abstract}
The calculation of the oscillation condition is one of the main points of oscillator analysis. Its determination in finite term allows one to calculate the steady state amplitude and frequency of the oscillator.

Symbolic solutions provide an additional insight into the behavior of the circuit. As an example the sensitivity of the oscillator to parameter change can be expressed in an exact form. Numerical solutions are not as helpful as symbolic solutions in the design stage.

We present a technique, based on the gyrator transformation, to set up the nonlinear equation network in a form suitable to be solved with analytical methods.

We develop a symbolic program based on this technique. As an example, the symbolic program is applied to compute the exact expression of the steady state frequency and amplitude of the Van der Pol oscillator and the Colpitts oscillator.
\end{abstract}

\section{Introduction}

Oscillators circuit generate the signals that are used as the time or phase reference. There are essential components in almost any electronic circuit.

Our aim is to build a symbolic program dedicated to the analysis of oscillators. There are many reasons why one may be interested in symbolic results. In the oscillator special case the most important ones are as follows :

- Giving the exact contribution of a circuit element to the frequency or amplitude variation.

- Computing exactly the effect of a noisy element on the frequency stability.

Once the nonlinear equation system of the network is obtained, the finite term solution can be found by two different methods:

- Harmonic balance like method. Harmonic balance is well established as a solving method for nonlinear circuits driven by one or more periodic inputs. This method is suitable to study the steady state frequency and amplitude of the oscillation.

\footnotetext{
*LPMO-CNRS, 32 avenue de l'Observatoire, 25044 Besançon Cedex, France.ratier@ieee.org, brendel@1pmo.edu.

${ }^{\dagger}$ CNES, 18 avenue E. Belin, 31055 Toulouse Cedex, France.
}

- Volterra and Wiener theories of nonlinear systems. This method is based on the transformation of the nonlinear circuit into a sum of linear circuits [5][6]. Then, the resulting linear circuit can be analyzed by graph theoretical methods. This method is suitable to study the transient behavior of the oscillator.

The technique used to determinate the network equations must be chosen carefully with the previous solving method in mind. We choose to implement the gyrator transformation method which is mathematically equivalent to the modified nodal analysis technique but conceptually superior.

\section{Gyrator Transformation}

Most of the computer programs used to analyze electronic circuits are based on the well-known modified nodal analysis (MNA) method [7]. This method allows a straight and systematic generation of the network equation.

Nevertheless, we choose to implement the gyrator transformation method as a pre-process of the MNA method. The use of gyrator has been known for a long time by circuit theorists [4] but seems to be of little use in practice. Even so, the gyrator method has many advantages over the modified nodal analysis method.

- At the opposite of the modified nodal analysis, the gyrator method does not need a separate handling of the branch currents into the voltage (controlled or independent) sources. Each unknown current is replaced by an unknown voltage.

- Most important topological [1] (or graph theoretical) methods assume that the admittance matrix of a linear network contains only RLC elements and voltagecontrolled current sources (VCCS).

In the gyrator method, all circuit elements are converted into voltage-controlled current sources ${ }^{1}$. That is, all linear n-terminal network will contain only RLC and VCCS type elements.

- The calculation of frequency domain sensitivity is difficult to handle with the MNA method because many additional nodes must be added [1]. With the gyrator method all sensitivities are expressed with respect

\footnotetext{
${ }^{1}$ Any admittance can be seen as a VCCS where the controlled voltage is taken beetween the two node of the VCCS itself.
} 
to voltage-controlled current sources, consequently, no additional node is needed.

- From a programming point of view, the uniformity of the approach allows to write the symbolic program which sets up the network equation in a very concise way.

The key element of the gyrator-nodal method is the (negative) gyrator circuit shown in Fig. (1). The gyrator transforms branch currents into nodal voltages, the fundamental relation connecting both variables are :

$$
\begin{aligned}
& I_{1}=g V_{34} \\
& I_{3}=g V_{12}
\end{aligned}
$$

In the particular case where the node 4 is connected to ground, each gyrator adds one auxiliary node having the voltage with respect to ground being proportional to the current of the transformed branch.
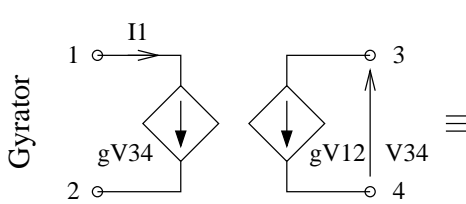

(a)

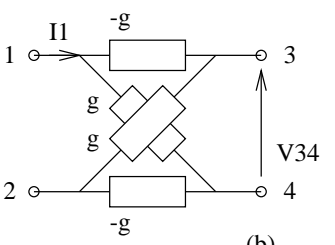

(b)
Figure 1. Negative gyrator circuit

The independent voltage source is expressed in terms of gyrator and VCCS under the form shown in Fig. (2).

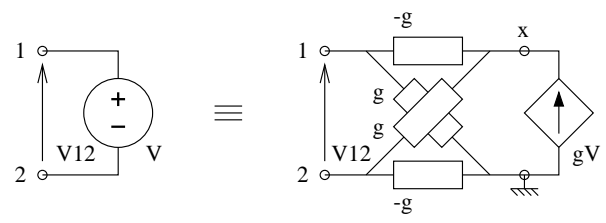

Figure 2. Equivalent circuit of independant source voltage

The equivalent circuit of the controlled-source ${ }^{2}$, not including VCCS, is represented in Fig. (3) next page.

The only admittance type which does not posses a direct admittance matrix is the mutually-coupled inductance. Its equivalent circuit is shown in Fig. (4).

The additional parameters $g: g_{1}, g_{2}, \ldots$ does not appear in the final solution. Consequently, they may be arbitrarily assigned any literal name or finite value, real or complex other than zero.

\begin{tabular}{l|l} 
VCVS & Voltage-Controlled Voltage Source \\
CCCS & Current-Controlled Current Source \\
VCCS & Voltage-Controlled Current Source \\
CCVS & Current-Controlled Voltage Source
\end{tabular}

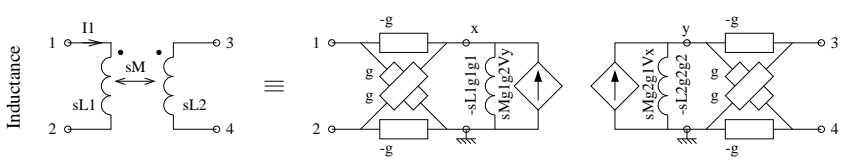

Figure 4. Equivalent circuit of mutually-coupled inductance

\section{Demonstrating Examples}

We apply our symbolic analysis program on two wellknown examples : the Van der Pol and the Colpitts oscillator.

\section{Van der Pol Oscillator}

Fig. (5) shows a Van der Pol oscillator. The resonator is reduced to its series resonant branch $\left(R_{q}, L_{q}, C_{q}\right)$. The nonlinear gain of the amplifier is

$$
V_{2}=A V_{1}\left(1-\epsilon V_{1}^{2}\right)
$$

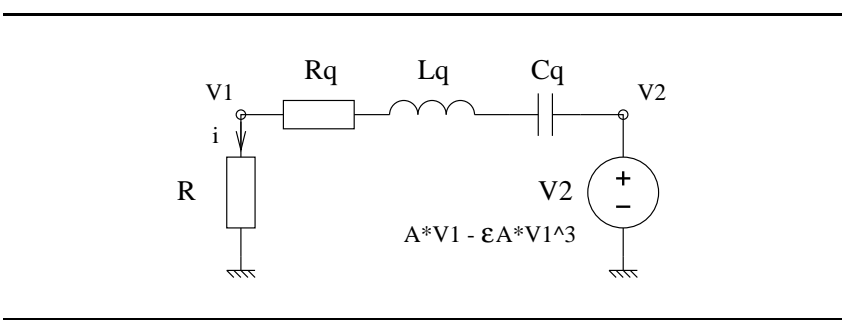

Figure 5. Van der Pol oscillator.

The symbolic program parses the Spice netlist of the circuit and determines the nonlinear literal expression of the voltage equations. This leads to the literal expression of the current $i(t)$ across $R$ (Eq. 4).

$$
\begin{aligned}
& A R \frac{d i(t)}{d t}-3 \epsilon A R^{3} i^{2}(t) \frac{d i(t)}{d t} \\
& -R \frac{d i(t)}{d t} \\
& -R_{q} \frac{d i(t)}{d t}-L_{q} \frac{d^{2} i(t)}{d t^{2}}-\frac{i(t)}{C_{q}}=0
\end{aligned}
$$

When using high-Q series resonant circuit, the loop current in the oscillator is almost perfectly sinusoidal. Using the technique of harmonic balance, the current $i(t)$ is assumed to have a solution of the form

$$
i(t)=I \cos (\omega t)
$$

This expression is substituted into Eq. (4). The equation resulting from the sine terms represents the frequency equation (Eq. 6).

$$
L_{q} \omega^{2}-\frac{1}{C_{q}}=0
$$



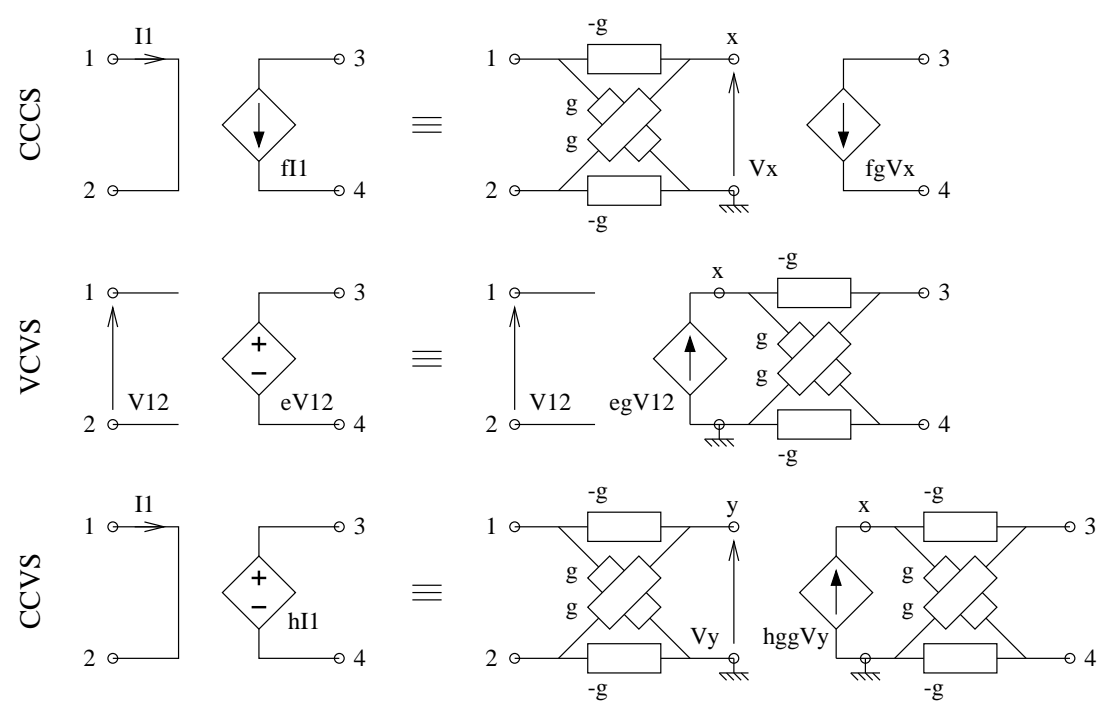

Figure 3. Equivalent circuit of controlled sources

The equation resulting from the cosine terms represents the amplitude equation (Eq. 7).

$$
\frac{3}{4} \epsilon A R^{3} I^{2}+R(1-A)+R_{q}=0
$$

The steady state frequency and amplitude $(\omega, I)$ obtained from Eq. (6) and Eq. (7) corresponds to those given in [2].

\section{Colpitts Oscillator}

Fig. (6) shows a simplified Colpitts oscillator already studied by Frerking [3]. The crystal is represented by its series resistance $R$ and an equivalent inductance $L$. To make the comparison easier, we used the same notation as Frerking.

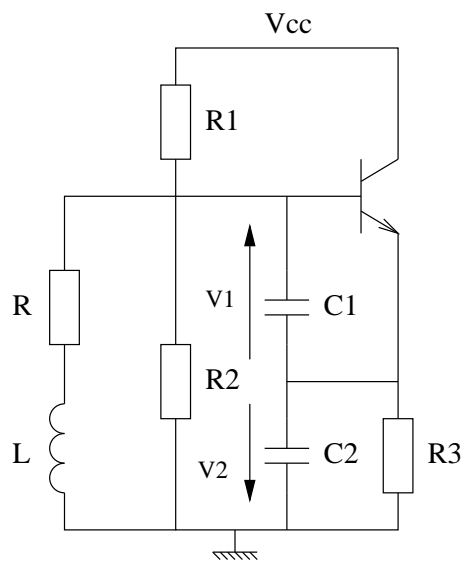

Figure 6. Colpitts oscillator.
The circuit of Fig. (6) is drawn again without the bias circuit in Fig. (7). The current $i_{b}$ is given by Eq. (8) where $V_{0}$ is the base bias voltage.

$$
i_{b}=I_{r} \exp \left[\frac{q}{K T}\left(V_{1}(t)+V_{0}\right)\right]
$$

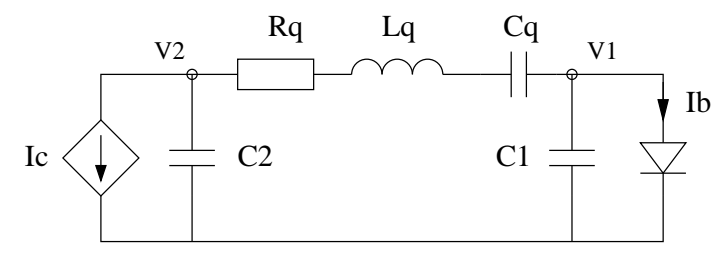

Figure 7. Equivalent circuit of Colpitts oscillator.

The symbolic program parses the Spice netlist of the circuit and determines the nonlinear literal expression of the voltage equation (Eq. 9) where $A\left(V_{1}\right)(t)=e^{\frac{q}{K T}\left(V_{1}(t)+V_{0}\right)}$.

$$
\begin{aligned}
& \frac{d V_{1}(t)}{d t}+\frac{d V_{1}(t)}{d t}\left\{\frac{R}{L}+\frac{1}{C_{1}}\left(\frac{q I_{r}}{K T}\right) A\left(V_{1}\right)(t)\right\} \\
& +\left(\frac{d V_{1}(t)}{d t}\right)^{2}\left\{\frac{I_{r}}{C_{1}}\left(\frac{q}{K T}\right)^{2} A\left(V_{1}\right)(t)\right\} \\
& +\frac{d V_{1}(t)}{d t}\left\{\frac{1}{L}\left(\frac{1}{C_{1}}+\frac{1}{C_{2}}\right)+\frac{R}{L C_{1}}\left(\frac{q I_{r}}{K T}\right) A\left(V_{1}\right)(t)\right\} \\
& +\frac{I_{r}(\beta+1)}{L C_{1} C_{2}} A\left(V_{1}\right)(t)=0
\end{aligned}
$$

The voltage $V_{1}(t)$ is assumed to have a solution of the form (Eq. 10).

$$
V_{1}(t)=E \cos (\omega t)
$$


This expression is substituted into Eq. (9). The terms under the forms $\exp (x \cos (\omega t))$ are expanded using the identity (Eq. 11) where $I_{m}(x)$ represents the modified Bessel function of the first kind and order $m$.

$$
\exp (x \cos (\omega t))=I_{0}(x)+2 \sum_{n=1}^{\infty} I_{n}(x) \cos (\omega t)
$$

The equation resulting from the sine terms represents the frequency equation (Eq. 12).

$$
\begin{aligned}
& \omega-\frac{1}{L}\left(\frac{1}{C_{1}}+\frac{1}{C_{2}}\right) \\
& -\frac{R}{L C_{1}}\left[I_{0}\left(\frac{q E}{K T}\right)-I_{2}\left(\frac{q E}{K T}\right)\right]\left(\frac{q}{K T}\right) I_{r} e^{\frac{q V_{0}}{K T}}=0
\end{aligned}
$$

The equation resulting from the cosine terms represents the amplitude equation (Eq. 13).

$$
\begin{aligned}
& -\frac{R \omega^{2} E}{L}-\frac{\omega^{2}}{C_{1}}\left[I_{0}\left(\frac{q E}{K T}\right)+I_{2}\left(\frac{q E}{K T}\right)\right]\left(\frac{q E}{K T}\right) I_{r} e^{\frac{q V_{0}}{K T}} \\
& +\frac{\omega^{2}}{2 C_{1}}\left[I_{1}\left(\frac{q E}{K T}\right)-I_{3}\left(\frac{q E}{K T}\right)\right]\left(\frac{q E}{K T}\right)^{2} I_{r} e^{\frac{q V_{0}}{K T}} \\
& +\frac{2(\beta+1)}{L C_{1} C_{2}} I_{1}\left(\frac{q E}{K T}\right) I_{r} e^{\frac{q V_{0}}{K T}}=0
\end{aligned}
$$

The steady state frequency and amplitude $(\omega, E)$ are obtained from Eq. (12) and Eq. (13). These results were obtained by Frerking [3] with considerable manipulations. The used of symbolic program with a suitable nodal method and resolution process finds again the same result, but above all gives the possibility to study much more complex circuits.

\section{Conclusion}

The symbolic program we present in this paper allows to determine the nonlinear network equation in a very uniform way. It implements the gyrator transformation method which has many advantages over the classical modified nodal analysis method.

Our program gives the finite term expression of the steady state oscillation amplitude and frequency directly from the Spice description netlist of the oscillator.

We compare our result on the Van der Pol and Colpitts oscillator for which the steady state frequency and amplitude can be obtained by hand after considerable manipulations.

In the case of more complex oscillators, which involve more components, the finite term solution expression could be inextricable. Even so, it is possible to obtain the exact dependency of one or a few circuits component by substituting all the other component names by their numerical value.

Now we will focus on the implementation of the Wiener method, where the very nice property of the gyrator transformation will be fully exploited.

\section{Acknowledgments}

This work has been supported by CNES under contract: \# 714/CNES/99/7671/00.

\section{References}

[1] L. O. Chua, P.-M. Lin, Computer-Aided Analysis of Electronic Circuits, Prentice-Hall (1975).

[2] R. Brendel, D. Gillet, N. Ratier, F. Lardet-Vieudrin, J. Delporte, Nonlinear dipolar modelling of quartz crystal oscillators, Proc. of 14th EFTF (2000).

[3] M.E. Frerking, Crystal oscillator design and temperature compensation, Van Nostrand Reinhold (1978).

[4] H. Kremer, Numerical Analysis of Linear Networks and Systems, Artech House (1987).

[5] M. Schetzen, Multilinear Theory of Nonlinear Networks, Journal of the Franklin Institute, Vol. 320, No. 5., pp. 221-247, November 1985.

[6] M. Schetzen, The Volterra and Wiener theories of nonlinear systems, Krieger Publishing Company (1989).

[7] J. Vlach, K. Singhal, Computer Methods for Circuit Analysis and Design, Van Nostrand Reinhold (1983). 